\title{
A new global marine magnetic anomaly data set
}

\author{
Y. Quesnel, ${ }^{1,2}$ M. Catalán, ${ }^{3}$ and T. Ishihara ${ }^{4}$ \\ Received 8 October 2008; revised 26 January 2009; accepted 17 February 2009; published 28 April 2009.
}

[1] The Geophysical Data System (GEODAS) stores more than 20 million magnetic measurements acquired over oceans and seas since the 1950s. Usually, the original total field (TF) and magnetic anomaly values are both stored. The anomaly results from the subtraction of the core and external magnetic field estimates from TF values. The most recent International Geomagnetic Reference Field models available at the time of the surveys were used to estimate the core field component (these models were revised later). External fields were estimated from magnetic observatory data. However, most of the measurements were not corrected for the external fields. Here we use comprehensive models to properly remove the core and external magnetic fields from all original TF measurements stored in the GEODAS. Besides, a track-by-track analysis of each data is necessary mainly to correct or to remove many shifted values as well as to reduce the noise in some track lines. Two additional processes are applied to obtain a data set coherent over the world. It includes an adjustment of long-wavelength magnetic anomalies using the National Geophysical Data Center (NGDC) -720 model, plus a line leveling method which mainly reduced some inconsistencies between different surveys. The root mean square of the crossover differences was reduced from 179.6 to $35.9 \mathrm{nT}$. Comparisons of magnetic anomaly maps before and after our treatment also highlight an improvement in the quality and the coherence of the data set. This study will serve to build a new World Digital Magnetic Anomaly Map.

Citation: Quesnel, Y., M. Catalán, and T. Ishihara (2009), A new global marine magnetic anomaly data set, J. Geophys. Res., 114, B04106, doi:10.1029/2008JB006144.

\section{Introduction}

[2] The International Association of Geomagnetism and Aeronomy (IAGA) Task Group of the World Digital Magnetic Anomaly Map (WDMAM) pursues to produce a world map reflecting lithospheric magnetic field anomalies at 5 $\mathrm{km}$ of altitude. Priority is given to near-surface data, such as ground, airborne and marine magnetics. In the first printed version of this map [Korhonen et al., 2007], empty oceanic areas were filled in by predictions from a lithospheric magnetization model. Over empty continental areas, as well as empty oceanic areas where the latter predictions do not exist, anomalies of the downward continued MF-5 magnetic field model [Maus et al., 2007a] remained. Indeed, this model was used to overprint all long-wavelength magnetic anomalies worldwide.

[3] Over the oceans, much of the magnetic data are stored in the Geophysical Data System (GEODAS) [Metzger and Campagnoli, 2007] of the U.S. National Geophysical Data

\footnotetext{
${ }^{1}$ CEREGE, UPCAM, Aix-en-Provence, France.

${ }^{2}$ Helmholtz Centre Potsdam GFZ German Research Centre for Geosciences, Section 2.3 Earth's Magnetic Field, Potsdam, Germany.

${ }^{3}$ Geophysics Department, Real Instituto y Observatorio de la Armada, San Fernando, Spain.

${ }^{4}$ Institute of Geology and Geoinformation, Geological Survey of Japan, AIST, Tsukuba, Japan.
}

Copyright 2009 by the American Geophysical Union. 0148-0227/09/2008JB006144\$09.00
Center (NGDC), which also stores, among others, bathymetric and gravity data. To our knowledge, the quality of these data sets have not been checked at a global scale until the recent study of Chandler and Wessel [2008]. On the basis of a statistical analysis, the authors described a useful method to check, and therefore, to improve the quality of these data sets. Concerning magnetics, they indicated that magnetic anomalies will need to be recomputed with the latest IGRF models, and created an errata file mechanism to distribute point corrections. Indeed, such corrections are necessary since geomagnetic field models used to calculate marine magnetic field anomalies are usually inconsistent. Furthermore, detailed reports of external field and baseline corrections are rarely provided. Among various sources of errors, such as the instruments themselves, the data set also contains contributions due to the secular variation, which is unwanted for mapping the magnetic field of the oceanic lithosphere. Many spikes were also included in the marine data set used for the WDMAM. Additionally, incoherences between different surveys were not corrected, affecting the interpolation of the final grid over oceanic areas. The recent release of the comprehensive model CM4 [Sabaka et al., 2004] enabled to correct these total field (TF) marine data by a proper separation of spatial and temporal variations. It offered a good opportunity to check all data and to level different surveys. Besides the obvious benefit for the whole scientific community, these improvements should help to produce a new version of WDMAM. 


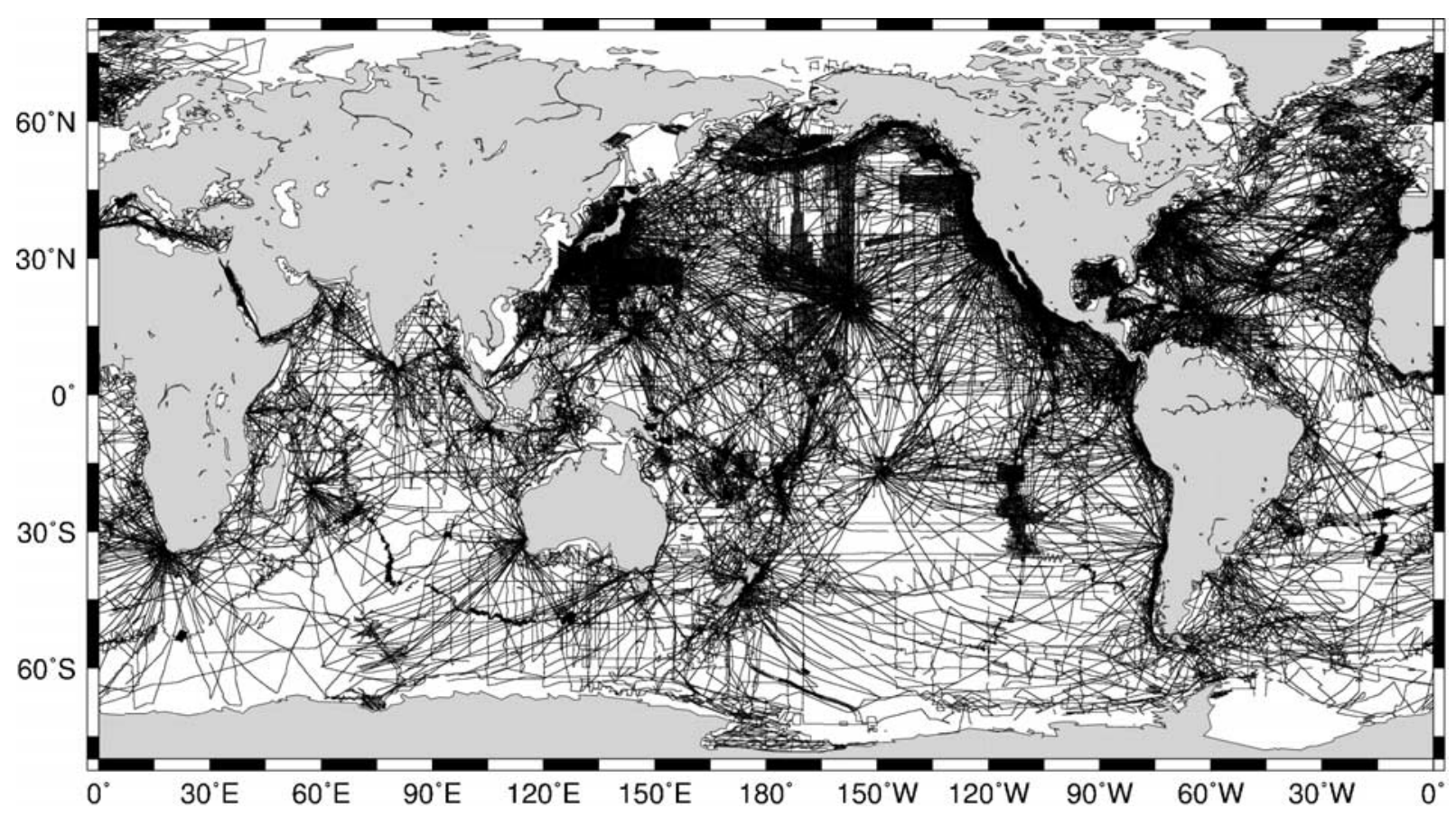

Figure 1. World marine magnetic surveys used in this paper. Acquisition times span from 1953 to 2003. This map shows the lack of measurements in the Southern Hemisphere oceans.

[4] In section 2, we introduce the data used for this study. The components estimated to reveal the magnetic anomaly are listed in section 3, highlighting their associated contributions to the error budget. In section 4, the processing method to resolve these problems is described, and the approach used to improve the coherence of the data set is detailed in section 5. Results are presented at the end of each of these sections. Some aspects of our method are then discussed in section 6, with emphasis on the leveling process.

\section{Marine Magnetic Data}

[5] Acquiring magnetic measurements onboard marine vessels for scientific purpose started in the 1950s. Much of the data acquired since then is stored in the GEODAS server. In this study, all marine magnetic measurements of the GEODAS DVD Version 5.0.10 were considered. This data set consists of 2411 cruises (Table 1), with acquisition times spanning from 1953 to 2003 . Each cruise contains either TF or anomaly values or both (for most of the cruises). Figure 1 shows the global distribution of these marine magnetic surveys. The northern Atlantic and Pacific oceans are extensively surveyed, whereas the southern oceanic regions display large gaps in magnetic measurements. Coastal areas as well as regions of tectonic or topographic anomalies, e.g., oceanic ridges, are usually well covered. This clearly indicates that marine magnetic data are heterogeneously distributed, and thus affecting the assessment of the global quality of this data set. Crossover analyses [Wessel and Watts, 1988; Hsu, 1995; Thakur et al., 1999] are here used. The anomaly crossover difference (COD) between two tracks should reflect the error budget. These errors are mainly due to temporal (i.e., secular) variations of the geomagnetic field, to external field con-

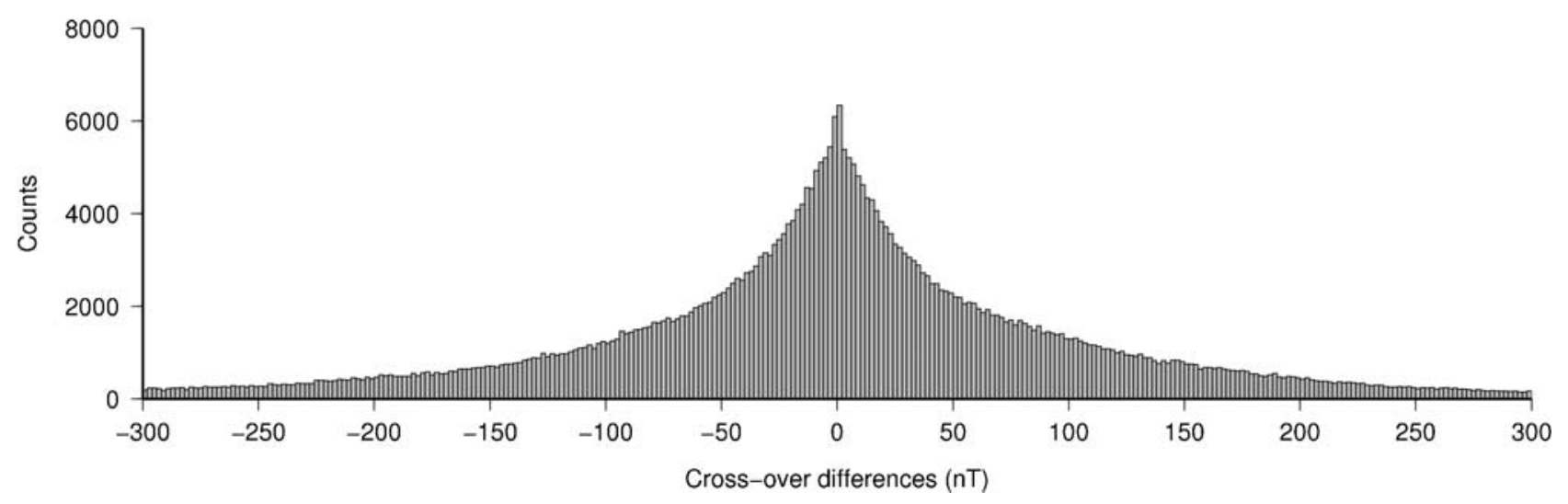

Figure 2. Histogram showing the distribution of magnetic anomaly differences at crossovers for the original data set. The range was arbitrarily limited to $\pm 300 \mathrm{nT}$, but larger differences exist (see text for details). 
Table 1. Magnetic Survey Cruises in GEODAS Version 5.0.10

\begin{tabular}{lc}
\hline \multicolumn{1}{c}{ Case } & Number of Cruises \\
\hline Original data set & 2411 \\
After 2002.5 (out of the time range of CM4) & \\
ODP205JR ODP206JR ODP207JR & 3 \\
ODP208JR ODP209JR ODP210JR & \\
Removed due to erroneous navigation data & 6 \\
PEGASUS POL6725a TBD375 & \\
Removed due to curious variation of total & \\
field and anomaly values & 8 \\
L380NP L480WG LEG177JR ODP203JR & \\
S169BS SI343912 & \\
Double counted & \\
E881SP = EVA800 & \\
F187BS $=$ FARN0687 & \\
F387AA $=$ FARN0887 & \\
GH7901 $=$ G179NP & \\
NBP93-7 = NBP93-8 & \\
POL7001 = P170WG & \\
POL7106 $=$ P671WG & \\
PZGSCXUS $=$ P172WF & \\
Removed by line leveling and visual & \\
check of magnetic anomaly maps & \\
CAPH-BHO DELP87T2 JPYN02BD & \\
L676AR SILS03BT V2007 & \\
Removed because of too short length & \\
INSV01WT L182NC RAPA00WT & \\
Remaining cruises (after line leveling) & \\
\hline aRemoved cruise. & \\
bData merged into the former cruise. & \\
\hline
\end{tabular}

tributions and to navigation errors. Figure 2 shows the distribution of all CODs for the original data set in the range $\pm 300 \mathrm{nT}$. About $9 \%(38,439)$ of CODs are out of range. The histogram reveals that about 72,56 , and $35 \%$ of the crossovers exhibit differences higher than 25,50 , or $100 \mathrm{nT}$, respectively. Our study aims to reduce these percentages. The root mean square value of all differences at all crossovers, $\mathrm{RMS}_{\mathrm{COD}}$, is equal to $179.6 \mathrm{nT}$. This $\mathrm{RMS}_{\mathrm{COD}}$ value will be used as a reference to estimate the global quality of the data set at each step of our study.

\section{Unwanted Signal Contributions}

[6] Because a single TF magnetic measurement is the sum of several contributions: core, crustal and external fields as well as instrumental noise, the crustal field estimation is subject to various perturbations. The main sources are (1) imprecise ship position, (2) instrumental errors, (3) incomplete cancellation of external field contributions, and (4) incomplete removal of core field contribution. Each source is considered in detail below.

\subsection{Position of the Ship}

[7] Before the 1970s, it was difficult to obtain a positioning accuracy better than $1 \mathrm{~km}$ because celestial navigation was the only available method outside radio navigation network areas. This situation improved significantly with the Doppler satellite technique, which provides an accuracy of several hundred meters. When Global Positioning Systems (GPS) became available in the early 1990s, errors on positioning were reduced to less than $100 \mathrm{~m}$ and, since 1 May 2000, to less than $20 \mathrm{~m}$ worldwide. Since the GEODAS data set covers a wide period of time, positioning accuracy varies.
[8] Usually, magnetometers were towed a few hundred meters astern ships. As this information was not available for most of the surveys, no position correction due to this towing shift was made in our study. This error could have an impact, which is difficult to assess in areas of high magnetic gradient.

\subsection{Instrumental Errors}

[9] Most of marine surveys (even in the 1950s) use proton-precession or optically pumped magnetometers because fluxgate sensors are not suitable onboard ships. Nowadays, proton-precession magnetometers provide an accuracy up to $0.1 \mathrm{nT}$, with drifts of up to $0.05 \mathrm{nT} / \mathrm{a}$ [Sapunov et al., 2001]. Comparisons between old and modern generation of magnetometers revealed an accuracy always better than $2 \mathrm{nT}$ and an internal precision of about 0.1 nT [Váczyová and Vörös, 2001]. Since the noise may depend on each cruise, no accurate estimation and so, no removal, was possible. Only the leveling phase using tie point residuals may reduce instrumental error budget for the final data set.

\subsection{Estimation of the External Magnetic Field}

[10] External field contributions could be estimated during the survey by using reference stations, e.g., magnetic observatories. Even if reference station data were available, they often lack precision because stations are not located close enough to the surveyed area. Indeed, most of the GEODAS marine magnetic data are not corrected of external fields [Chandler and Wessel, 2008].

\subsection{Estimation of the Core Magnetic Field}

[11] The International Geomagnetic Reference Field (IGRF) models of the survey period were commonly used to remove core field contribution from TF measurements. These models consist of spherical harmonic coefficients that predict the main field and its secular variation over a 5-year interval [Macmillan and Maus, 2005]. As they lack accurate predictions, a posteriori revision is needed to generate the so-called DGRF (Definitive Geomagnetic Reference Field). Nevertheless, to estimate the core field during or just after a survey, scientists had to use IGRF models. In our data set, the use of different IGRF models leads to inconsistencies between nearby surveys of different epochs.

\subsection{Other Sources of Errors}

[12] An inaccurate estimation of acquisition time can be another source of error. Most of these errors are easily identified, e.g., time in reverse order, or constant for a large number of measurements. Additionally, errors can occur during TF measurement handling, especially in old surveys for hand-written records. Details and examples are given in Section 4.4.

\section{Data Cleaning}

[13] The first part of the processing consisted in a thorough check of the data, track by track in order to modify or remove wrong entries, if necessary. The data were graphically inspected, by displaying magnetic anomalies along each track together with bathymetry, ship speed and the first derivative of acquisition time. To avoid the previously listed errors, modifications consisted of five 

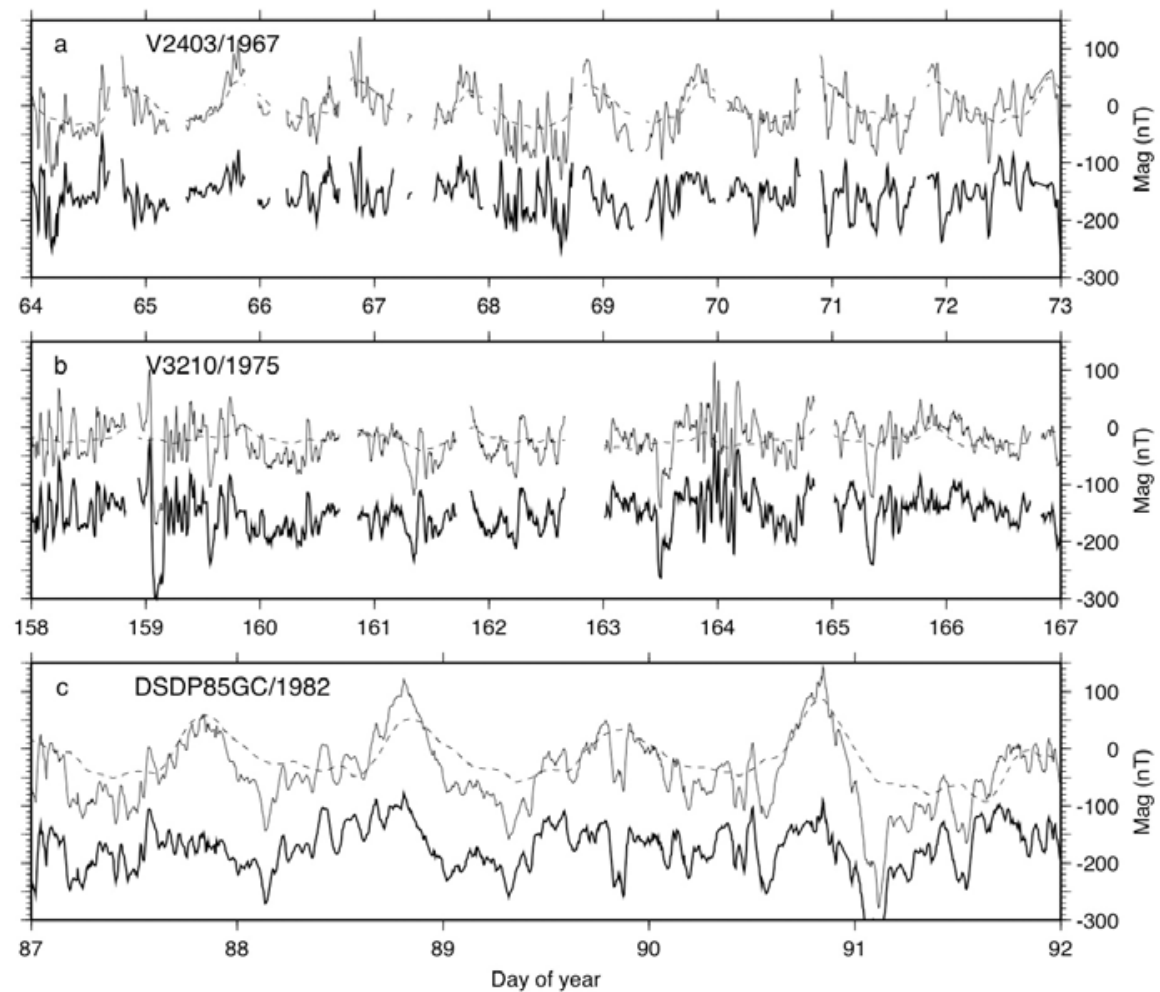

Figure 3. Magnetic anomaly profiles along three E-W track lines of different epochs in the equatorial Pacific area. Fine and thick lines show the original and corrected magnetic anomaly profiles. Thick curves are shifted by $-150 \mathrm{nT}$ for clarity purpose. The external field correction given by the CM4 model is shown by the dashed line over the original magnetic anomaly curve.

separate editing steps: (1) correction of time and position errors, (2) removal of core and external field contributions, (3) removal of erroneous cruise data, (4) correction/ removal of outliers, jumps and other transcription errors, and (5) correction/removal of noisy data. All these modifications compose a process denoted as "cleaning" in sections 4.1-4.6.

\subsection{Correction of Time and Position Errors}

[14] For each track, the acquisition time of the measurements was previously checked. Records with duplicate or decreasing time were deleted, whereas obvious errors in time recording were corrected. For example, all surveys carried out by the Hydrographic Department of Japan (HDJ) before 1982 had incomplete time information, and, sometimes, the date was missing. Finally, the records did not seem to be sequential. In this case, time information was filled in by using available cruise reports and track maps as reference. The accuracy of this method was, in the worst case, within 1 month of the true date, which is acceptable to estimate main field values.

[15] In order to check navigation data, vessel speed was calculated using the gradient of distance versus the time difference between consecutive data. Although $99 \%$ of the computed speeds were less than 16.3 knots [Chandler and Wessel, 2008], some exceeded 20 knots without serious navigation errors, because of small errors in position and time estimations for short periods (1 to $5 \mathrm{~min}$ ). Hence, consecutive data showing a cruise speed higher than 50 knots were removed. If one or a few erroneous navigation estimations were detected along a track, they were recalculated by simple interpolation of surrounding values. However, in a given cruise, if five or more consecutive data presented navigation errors, or if 10 or more data segments showed such errors, corresponding records were removed.

\subsection{Removal of Core and External Fields}

[16] The Comprehensive Model 4 (CM4) [Sabaka et al., 2004] was designed using POGO, Magsat, Oersted, and CHAMP measurements, as well as observatory data to account for magnetic sources and their temporal variations from 1960 to July 2002. The CM4 is more accurate than IGRF models to estimate (and here, to remove) the core field for this period. Moreover, it provides a proper estimate of external field contributions anywhere in the world, which is very valuable for marine data far from magnetic observatories.

[17] For the 6 cruises after 2002.5, the CHAOS model [Olsen et al., 2006] was used to remove the core field. The resulting data displayed curious trends of magnetic anomalies ranging from $-10,000 \mathrm{nT}$ to $+16,000 \mathrm{nT}$, suggesting instrumental errors. Considering their short lengths, these cruises were removed. For the 5 cruises prior to 1960, the spherical harmonic coefficients corresponding to this period in the IGRF-10 model [Macmillan and Maus, 2005] were used to estimate the core field. No correction of external field was made.

[18] Figure 3 shows magnetic anomaly profiles along three track lines of 1967, 1975, and 1982. These tracks 


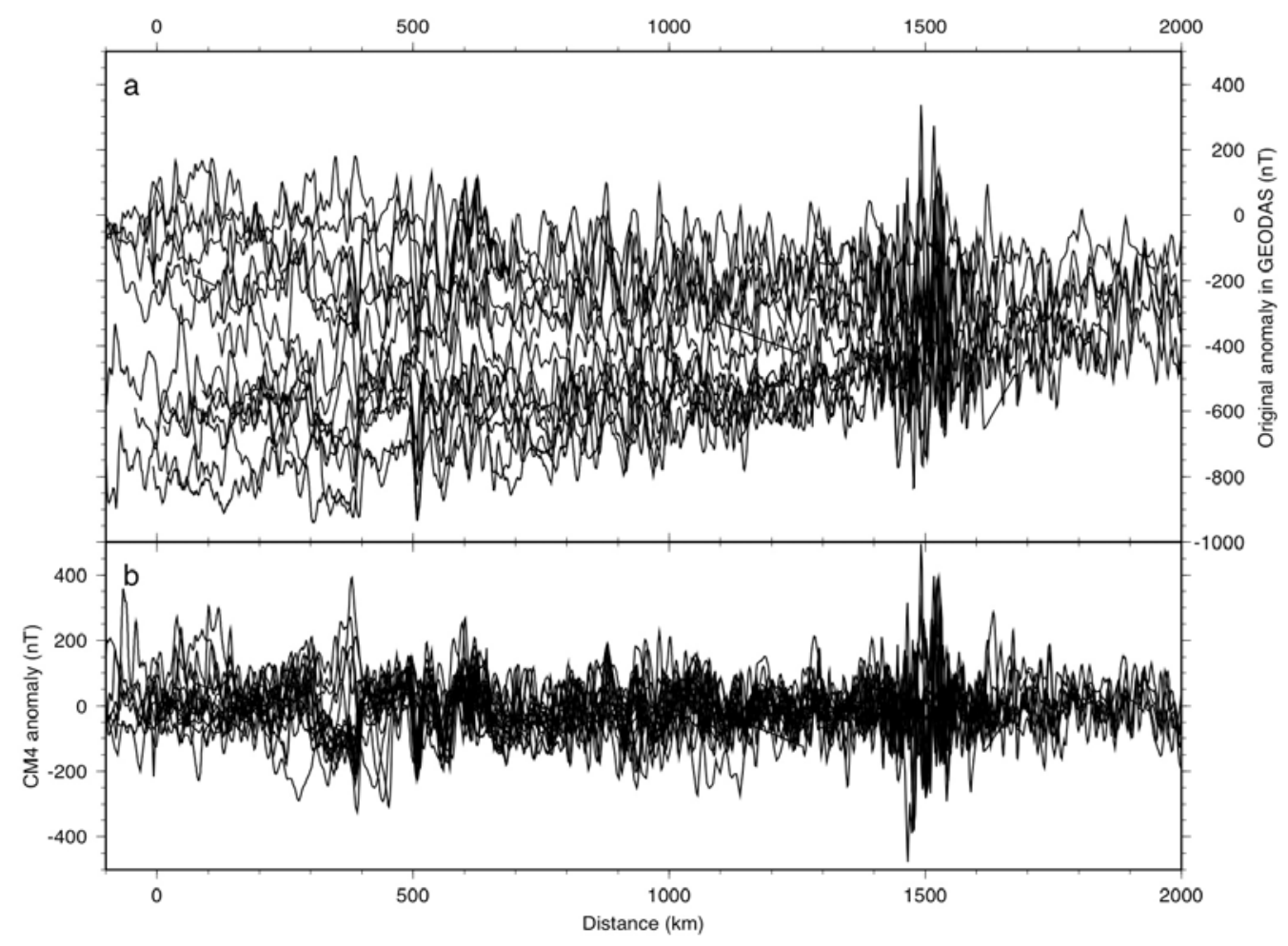

Figure 4. Magnetic anomaly profiles along 18 parallel and neighboring cruises over the western part of the North Atlantic. (a) Original GEODAS anomaly values. (b) The CM4-corrected values. The 18 cruises were carried out at different epochs between 1964 and 1997. Large anomaly values at about $1500 \mathrm{~km}$ of distance are due to the crossing of the North Atlantic spreading axis.

head westward in the east Pacific, from about 110 to $150^{\circ} \mathrm{W}$ between 0 and $5^{\circ} \mathrm{N}$. The observed profiles clearly show daily variations of different amplitudes: intermediate in 1967 (Figure 3a), small in 1975 (Figure 3b), and large in 1982 (Figure 3c). The external fields predicted by the CM4 model coincide well with these variations, and, after correction, daily variations are almost not visible in the magnetic anomaly profiles. Thus, the use of the CM4 is clearly efficient for removing external field contributions, displaying more precisely the crustal magnetic field.

[19] Similarly, Figure 4 shows the magnetic anomaly profiles of eighteen parallel and neighboring cruises over the North Atlantic before (Figure 4a) and after (Figure 4b) applying the CM4 correction. These tracks were acquired at different epochs between 1964 and 1997. The original profiles have values ranging from -900 to $200 \mathrm{nT}$, whereas after CM4 corrections, anomalies are of smaller amplitude, within $\pm 200 \mathrm{nT}$. This improvement is mainly due to a better estimation of the main field secular variation during the corresponding 1964-1997 period. It illustrates the high accuracy of the CM4 model to extract the core field from the original GEODAS anomalies.

\subsection{Removal of Erroneous Cruise Data}

[20] Several cruises were removed from the original data set (Table 1). Some, because they show strange variations of $\mathrm{TF}$ and anomaly values (e.g., constant increase or decrease for long periods, variation proportional to latitude values, etc.). Others, because the cruise data were associated to obvious navigation errors, as illustrated by the POL6725 cruise, where some data were located on land in the Mozambique area. Similarly, cruise TBD375, carried out by the South African Geological Survey, exhibits tracks crossing the Palawan Island (Philippines).

[21] Nevertheless, the GEODAS catalogue may still contain undetected double entries of cruises (Table 1). As a consequence, fictitious crossovers between twice cataloged cruises seem to exist, affecting the leveling processes and possibly giving incorrect COD results. The associated error depends on the number of crossovers of these cruises. So far, multiple entries of the same cruise data have been eliminated so that only one entry remains. When unifying the data set, we tried to keep as many magnetic data as possible. If two original cruises overlapped but did not match exactly in time, they were merged to cover the entire time period of both cruises. If sampling rates were different in each track, the one with the highest rate was kept.

\subsection{Correction/Removal of Outliers, Jumps, and Other Transcription Errors}

[22] Data outliers correspond to either instrumental or transcription errors that occurred during data recording, e.g., swap of two digits in the anomaly value. For instrumental errors, the value was deleted. For transcription errors, it was possible to retrieve the original anomaly value. Such cases concern mainly data from the earlier surveys (1960s and 1970s), when readings were transferred from an analog to a digital form.

[23] Moreover, temporary magnetometer errors as well as incorrect writing caused offsets, jumps in the recording. 

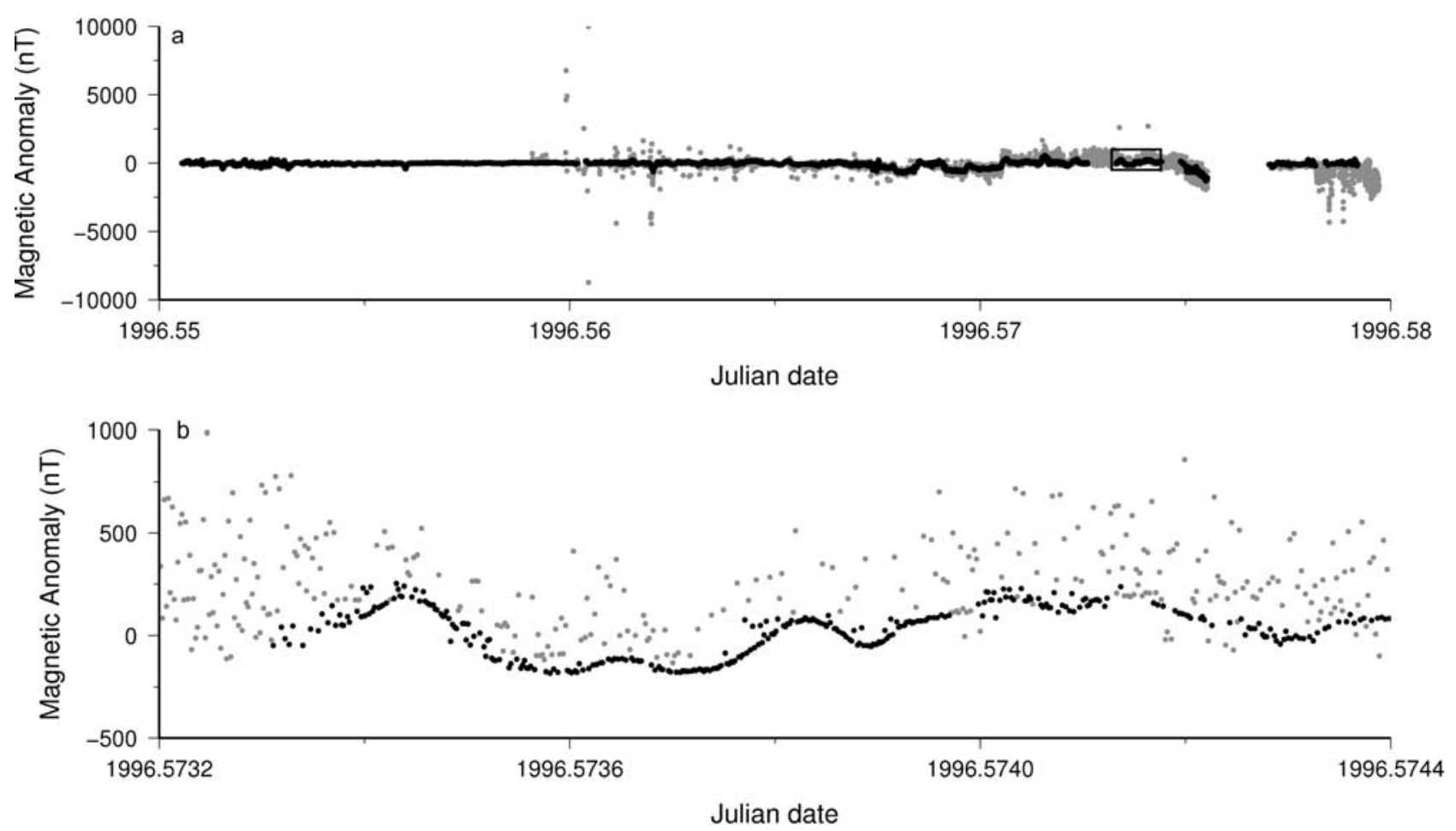

Figure 5. Example of a noisy track line. (a) Original magnetic anomaly profile (grey dots) and the same profile after extraction of spikes and low-pass filtering (black dots) are shown. The rectangle in Figure 5a specifies the area enlarged in Figure 5b. (b) Details of area indicated by rectangle in Figure 5a.

These errors occurred suddenly along a track and often correspond to jump values of 100, 200, 500, 1000, $2000 \mathrm{nT}$, etc. When detected, the values were corrected.

[24] Sampling a single magnetic anomaly with a different angle (e.g., change in the ship course) can also cause apparent jumps. The resulting offset is amplified when the sampling rate is slow. Bullard and Mason [1961] suggested that a ship can cause such magnetic disturbance, which varies with the ship heading. In our processing, such jumps were corrected if the offsets were greater than $50 \mathrm{nT}$. However, most of them were less than $10 \mathrm{nT}$.

\subsection{Correction/Removal of Noisy Data}

[25] Whereas for the previous cases a correction scheme can be applied, some data errors remain problematic. Large spikes outside $\pm 10,000 \mathrm{nT}$ were removed. However, some tracks do not show large spikes but rather show highfrequency variations in an acceptable range of amplitude. In such cases, a low-pass filter was applied to remove the short-wavelength variations unrelated to the crustal field. Furthermore, track lines considered too noisy after visual inspection were directly removed from the data set. Because of irregular sampling rates, this process could not be systematically applied to the whole data set but rather track by track. As an example, Figure 5a shows the filtered magnetic anomaly profile (in black) extracted from the original profile of the DELV02RR cruise (in gray). A zoomed view (Figure 5b) reveals how numerous filtered values (in black) are still incorrect. In such cases, i.e., when the result was not satisfactory, a point-by-point final extraction was performed.

[26] In order to distinguish the noise from the crustal signal, local bathymetry was also examined. Apparent noisy readings were not excluded if they were obtained in the proximity of the bedrock. Figure 6 illustrates a track line where high-amplitude anomaly values occurred when the ship passed several times over a seamount. If a seamount case was obvious, doubts (between true signal and noise) can still persist, particularly near the coasts. In these cases, measurements of neighboring track lines were checked to assess the regional coherence in the magnetic anomaly signal.

\subsection{Results of the Cleaning Phase}

[27] Figure 7 shows the distribution of all CODs with absolute values lower than $300 \mathrm{nT}$ for the CM4-corrected and cleaned data set. Compared to the distribution of the original data set (Figure 2), the improvement is clearly shown by the small range of differences $( \pm 150 \mathrm{nT})$ centered around zero. The percentages of $|\mathrm{COD}|$ values outside 300 , 100,50 , and $25 \mathrm{nT}$ are reduced from $9,35,56$, and $72 \%$ to $1,7,19$, and $53 \%$, respectively. The $\mathrm{RMS}_{\mathrm{COD}}$ for the whole data set decreased from 179.6 to $103.9 \mathrm{nT}$ (cases I and II, Table 2). When data prior to 1960 are removed, this value drops to $81.9 \mathrm{nT}$. This is probably due to the predictions of the IGRF-10 model [Macmillan and Maus, 2005] for 1950 and 1960, that are not as accurate as the CM4 predictions are for years after 1960 (see also section 5.1). Moreover, correction of the external field was not possible for these old cruises.

[28] In Figure 8, the magnetic anomaly map over North Atlantic is shown before (Figure 8a) and after (Figure 8b) the CM4 correction and cleaning phase. Original inconsistencies at long wavelengths mostly disappeared, reveal- 


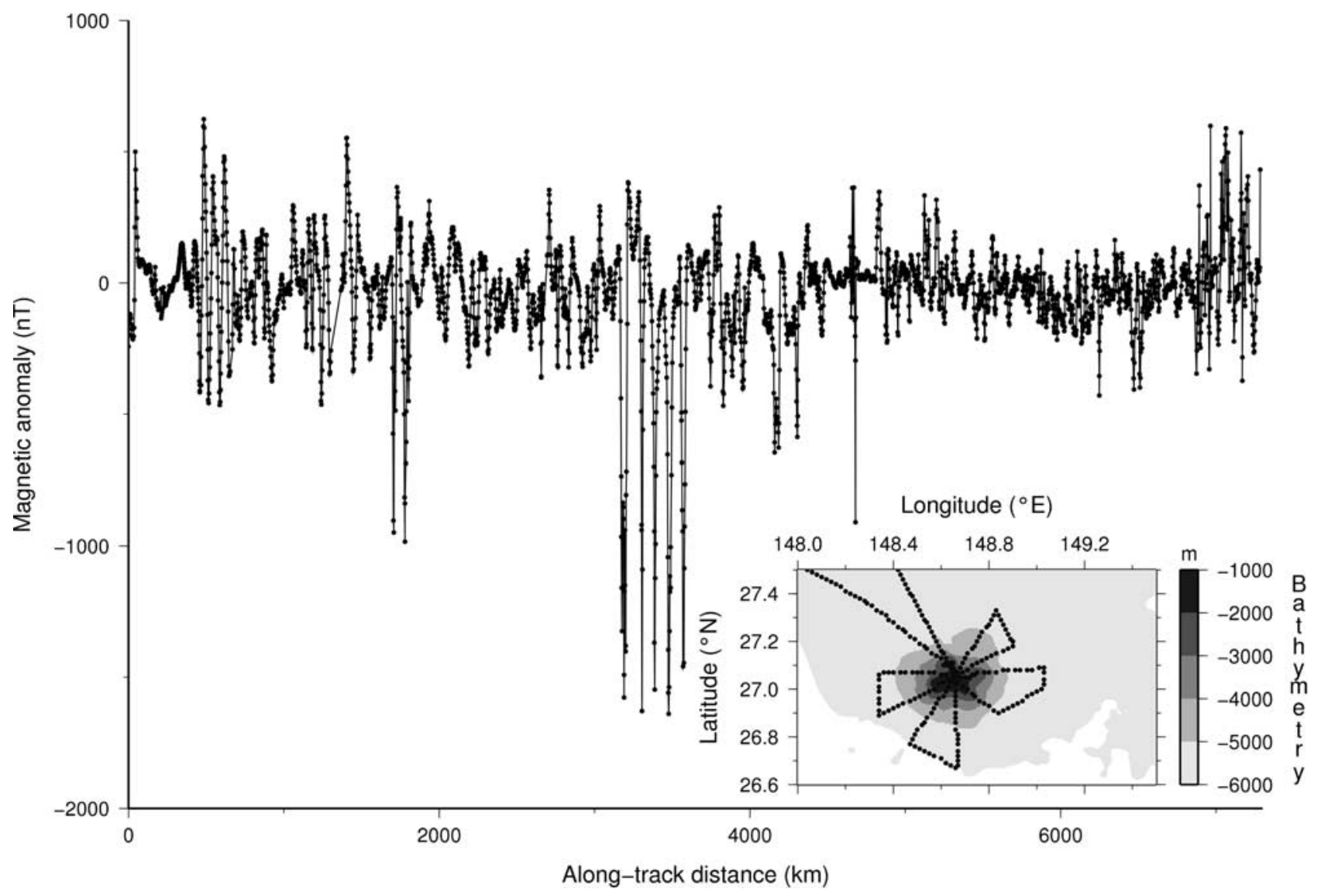

Figure 6. Example of an apparent noisy track line. Magnetic anomaly data were obtained during a nearly 1-month cruise performed in 1966 between 6 and 25 May in the northwestern Pacific Ocean. The sampling distance was $2.5 \mathrm{~km}$. The locations of the most anomalous measurements (between 3000 and $4000 \mathrm{~km}$ ) are shown in the inset, over a bathymetry map [Smith and Sandwell, 1997]. A seamount is clearly visible, explaining the large-amplitude and high-frequency variations of the corresponding magnetic signal.

ing the isochrons. Track lines with large anomaly values (mainly in red color) were also withdrawn.

\section{Application of NGDC-720 Crustal Field Model and Line Leveling}

[29] Some incoherences at different wavelengths between neighboring track lines still remain in the global data set and must be corrected. They can be due to various causes not detected previously: any kind of systematic offset or trending affecting a particular survey, magnetometer not properly calibrated, etc. The application of NGDC-720 model combined with a line leveling method unveils the global coherence of the CM4-corrected and cleaned data set.

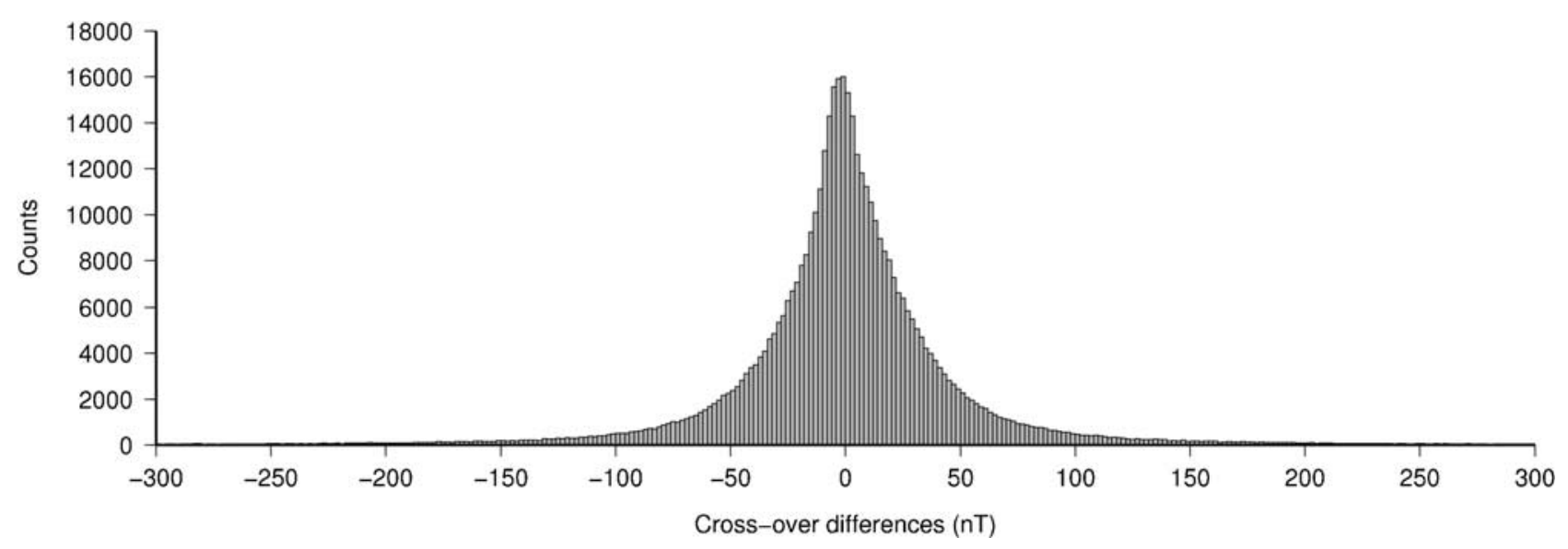

Figure 7. Same (except $\mathrm{Y}$ axis scale) as Figure 2 for the CM4-corrected and cleaned data set. 
Table 2. Summary of Results ${ }^{\mathrm{a}}$

\begin{tabular}{llcccl}
\hline Case & Number of Records & Number of CODs & Number of CODs $>300 \mathrm{nT}$ & $\mathrm{RMS}_{\mathrm{COD}}(\mathrm{nT})$ & \multicolumn{1}{c}{ Remarks } \\
\hline I & $20,260,076$ & 412,840 & 38,439 & 179.6 & Original data set $^{\mathrm{b}}$ \\
II & $19,911,022(-1.7 \%)$ & 425,487 & 6,079 & 103.9 & CM4-corrected and cleaned data set \\
III & $19,911,022(-0.2 \%)$ & 425,487 & 4,982 & 83.1 & NGDC-720 reduced \\
IV & $19,874,596(23,487$ & 4,161 & 78.4 & Data removal I \\
V & $19,874,596$ & 423,487 & 2,745 & 63.5 & Leveling (filter FW $=1000 \mathrm{~km})$ \\
VI & $19,874,596$ & 423,487 & 1,366 & 47.7 & Leveling (filter FW $=100 \mathrm{~km})$ \\
VII & $19,762,898(-0.6 \%)$ & 422,121 & 0 & 35.9 & Data removal II \\
\hline
\end{tabular}

aAbbreviations: COD, crossover differences; CM4, comprehensive model 4 [Sabaka et al., 2004]; NGDC-720, National Geophysical Data Center magnetic model up to degree 720 [Maus, 2008]; FW, full width of the Gaussian filter. See text for details.

${ }^{\mathrm{b}}$ The double counted cruises are included in the total number of records in the original data set, but they are excluded in the calculation of CODs.

\subsection{Application of NGDC-720 Crustal Field Model}

[30] In order to check the long wavelengths in the magnetic anomaly data set, a coherent global model of the lithospheric magnetic field should be used. However, the detection of localized deviations is not possible by comparison with global grids sampled along track, as Chandler and Wessel [2008] performed for gravity or bathymetry. On the other hand, the recent NGDC-720 crustal field model [Maus, 2008] provides an expansion of the lithospheric field from spherical harmonic degree 16 to 720 , corresponding to the wave band of $2500 \mathrm{~km}$ to $56 \mathrm{~km}$. It was built using the MF-5 model [Maus et al., 2007a] and all available ground, airborne and marine magnetic measurements. Maus [2008] showed the worldwide coherent behavior of this model. So, NGDC-720 values were subtracted from the CM4-corrected and cleaned data set. The resulting data set should mainly contain wavelengths shorter than $50 \mathrm{~km}$, and each cruise average should be close to 0 . Figure 9 shows the distribution of these averages, which seems to be Laplacian, centered around 0 . A total of 45 cruises have offsets higher than $100 \mathrm{nT}$. There, the cruise average was arbitrarily subtracted from the reduced anomaly values. In particular, four of the five cruises before 1960 were affected by this average subtraction, because they showed offsets greater than $390 \mathrm{nT}$. As described before, anomaly values for these cruises were calculated using the main field predictions given by the corresponding spherical harmonic coefficients of the IGRF-10 model [Macmillan and Maus, 2005]. Applying the subtraction allowed to correct large offsets and to reduce the $\mathrm{RMS}_{\mathrm{COD}}$ to $83.1 \mathrm{nT}$ (case III, Table 2).

\subsection{Data Set Modifications}

[31] A preliminary leveling calculation was carried out by the method described in section 5.3, and $\mathrm{RMS}_{\mathrm{COD}}$ values were calculated for each track line. Cruises with poor quality data were thus detected. For instance, cruises CAPH-BHO and L676AR had $\mathrm{RMS}_{\mathrm{COD}}$ greater than $800 \mathrm{nT}$ even after the leveling calculation. In addition, spurious anomalies along the tracks of four other cruises remained after leveling. These cruises, as well as three cruises with continuous track line lengths smaller than $10 \mathrm{~km}$ (listed in Table 1), were removed from the data set before actual line leveling. The $\mathrm{RMS}_{\mathrm{COD}}$ was then reduced to $78.4 \mathrm{nT}$ before the following step (case IV, Table 2).

\subsection{Line Leveling}

[32] The line leveling method consisted of three steps. First, the difference between the anomaly value at every along-track point and a weighted average value, obtained from all data (except those along the track) within $25 \mathrm{~km}$ of the considered point, was calculated. The weight $w$ was a function of the distance $r$ :

$$
w=\left[r_{0}^{2} /\left(r_{0}^{2}+r^{2}\right)\right]^{2}
$$

where a value of $2 \mathrm{~km}$ was adopted for the constant $r_{0}$ (see section 6). In the second step, all differences were low-pass filtered (see below) and weighted using a value for each point given by the sum of all surrounding data weights. Finally, the resulting correction value was added to the original value to obtain a high-pass filtered and leveled magnetic anomaly value at every along-track point.

[33] Iteration of this three-step process was necessary because the surrounding data also changed after each step. Several iterations were applied until the RMS difference between consecutive iterations was lower than $0.2 \mathrm{nT}$.

[34] The filtering was needed because the adjustment to the NGDC-720 crustal field did not resolve long-wavelength offsets, i.e., they still existed but were weaker. A Gaussian

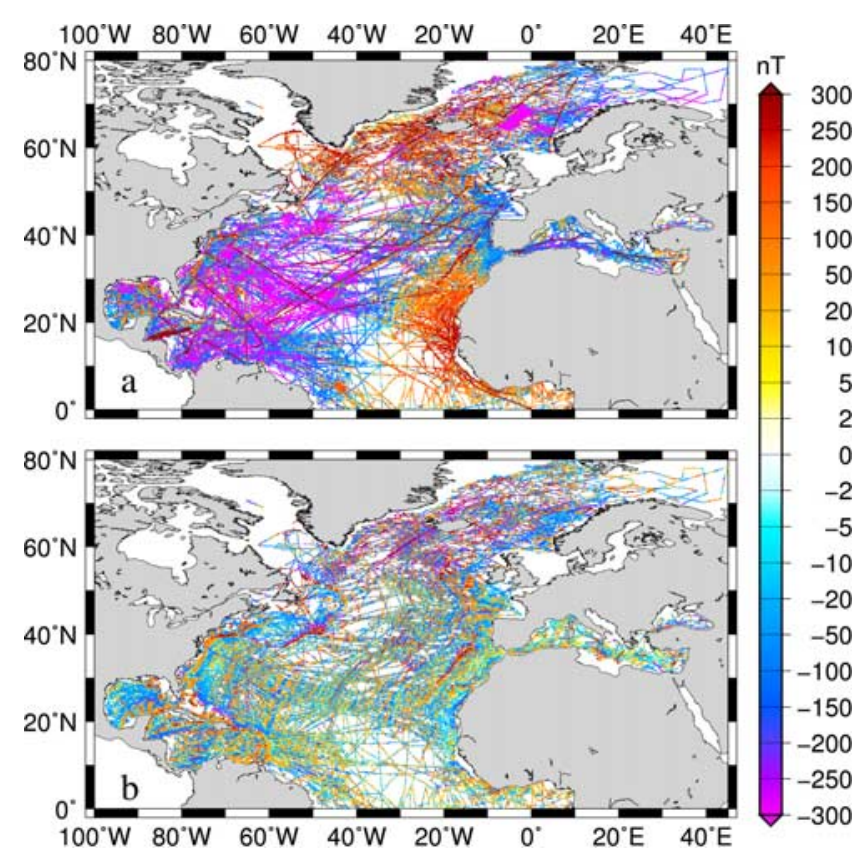

Figure 8. Magnetic anomaly maps over the North Atlantic, using (a) original anomaly values of the GEODAS data set and (b) CM4-corrected and cleaned values. 


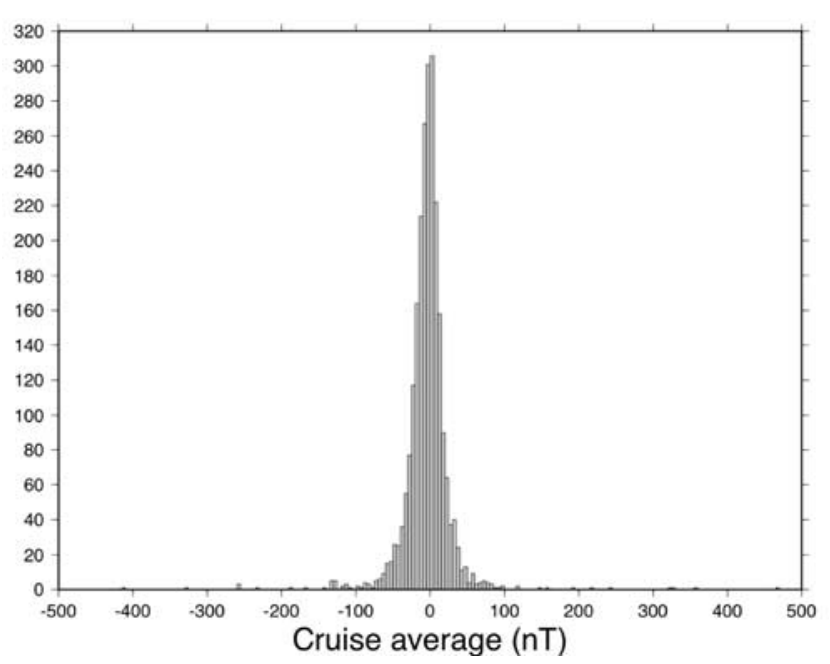

Figure 9. Histogram showing the distribution of the cruise averages of the CM4-corrected cleaned data after subtracting the NGDC-720 model [Maus, 2008].

filter was first used with a full width of $1000 \mathrm{~km}$. It allowed the smoothing of remaining offsets for isolated cruises. Consequently, the $\mathrm{RMS}_{\mathrm{COD}}$ improved to $63.5 \mathrm{nT}$ (case $\mathrm{V}$, Table 2). The same filter with a full width of $100 \mathrm{~km}$ was then applied to adjust shorter wavelengths. The $\mathrm{RMS}_{\mathrm{COD}}$ further improved to $47.7 \mathrm{nT}$ (case VI, Table 2). These two filtering steps are discussed in section 6.

\subsection{Last Modifications and Results of the Leveling Phase}

[35] The NGDC-720 values were added again to restore correct anomalies. The last data removal was then performed for data within 0.1 day of crossover points whose $|\mathrm{COD}| \mathrm{s}>300 \mathrm{nT}$ (only $0.6 \%$ of the total data). It provides a final $\mathrm{RMS}_{\mathrm{COD}}$ equal to $35.9 \mathrm{nT}$ (case VII, Table 2). The distribution of CODs is shown in Figure 10. Most values are now within $\pm 100 \mathrm{nT}$, following a Laplacian distribution centered around 0 . The percentages of $|\mathrm{COD}|$ values outside 25,50 , and $100 \mathrm{nT}$ are now 21, 9, and 3\%, respectively. In Figures 11 and 12, maps before (Figures 11a and 12a) and after (Figures $11 \mathrm{~b}$ and $12 \mathrm{~b}$ ) this leveling phase are shown over large (NW Pacific) and regional (NW Atlantic) areas, respectively. The large view highlights the improvements for long wavelengths, especially for anomalous straight features visible along several degrees of latitude and longitude. The regional view reveals that the inconsistencies between different surveys are considerably reduced or clearly disappeared. Thus, both short and wide oceanic magnetic anomalies (and the isochrons specifically) are better defined.

\section{Discussion}

[36] The cleaning phase was performed using a careful visual inspection of magnetic anomaly data along a track line. In some cases, the coherence with nearby track line values was also checked. To avoid errors due to individual (and perhaps, subjective) preferences, the global data set was split into three subsets, each was checked by an author. Once cleaned, the subset was cross-checked by the two other authors. Therefore, each subset was checked three times, reducing the influence of subjective decisions. One implication was that only few $(2.5 \%)$ data were removed.

[37] To build their NGDC candidate model for the WDMAM, Maus et al. [2007b] obtained a model correlation function with a correlation length of $15 \mathrm{~km}$, that fitted the former Soviet Union, the Australian and the North American magnetic anomaly grids. This means that two anomaly values separated by more than $15 \mathrm{~km}$ show little correlation. Anomalies in oceanic areas have probably different anisotropic characteristics: the correlation length across strike might be as large as $15 \mathrm{~km}$, and is likely to be larger than $15 \mathrm{~km}$ along strike. Therefore, the range of distance for the average calculation used to level the global marine magnetic data set could be restricted within $15 \mathrm{~km}$ across strike but a larger distance along strike. In our leveling method, the average calculation depends on the weight function described in equation (1), which decreases slowly from 1 to 0.5 up to the distance of $r_{0}$. Then, it decreases rapidly to zero afterward, so that the closest data and those within $r_{0}$ mainly contribute to the average. Furthermore, this weight function should not affect the fit at the crossovers. Several tests revealed that a value of $2 \mathrm{~km}$

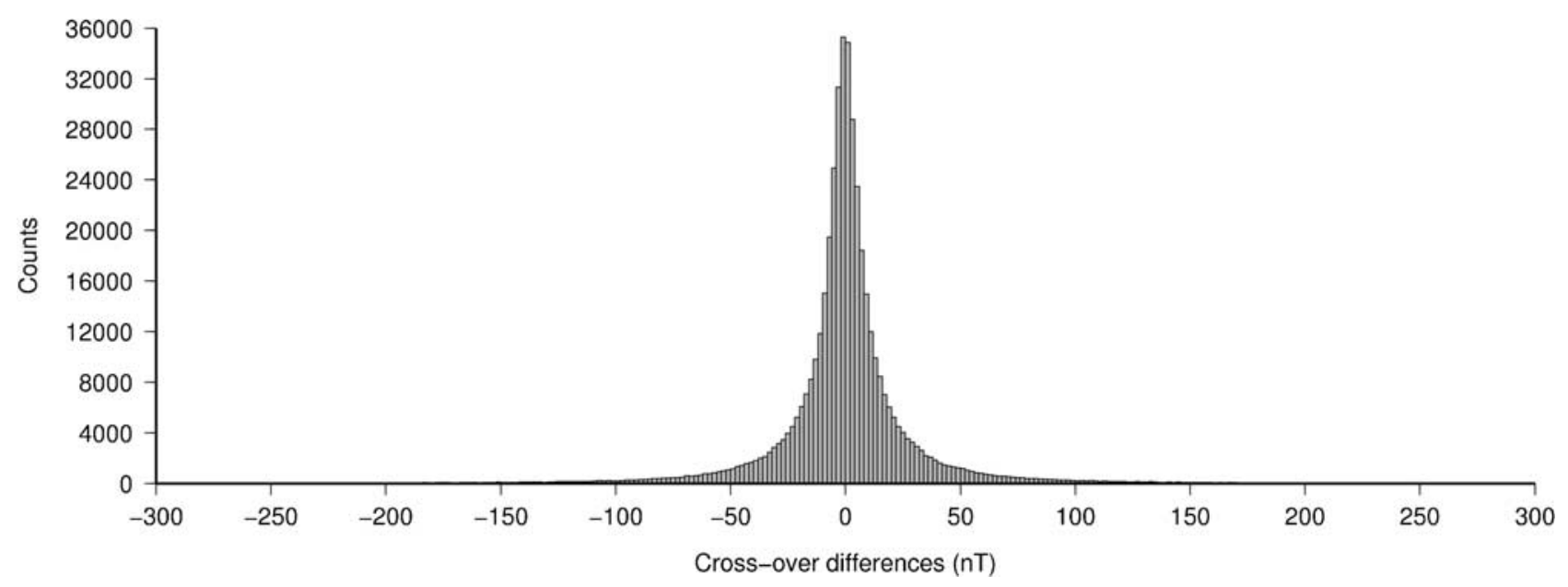

Figure 10. Same (except $Y$ axis scale) as Figure 2 for the CM4-corrected, cleaned, and leveled data set. 


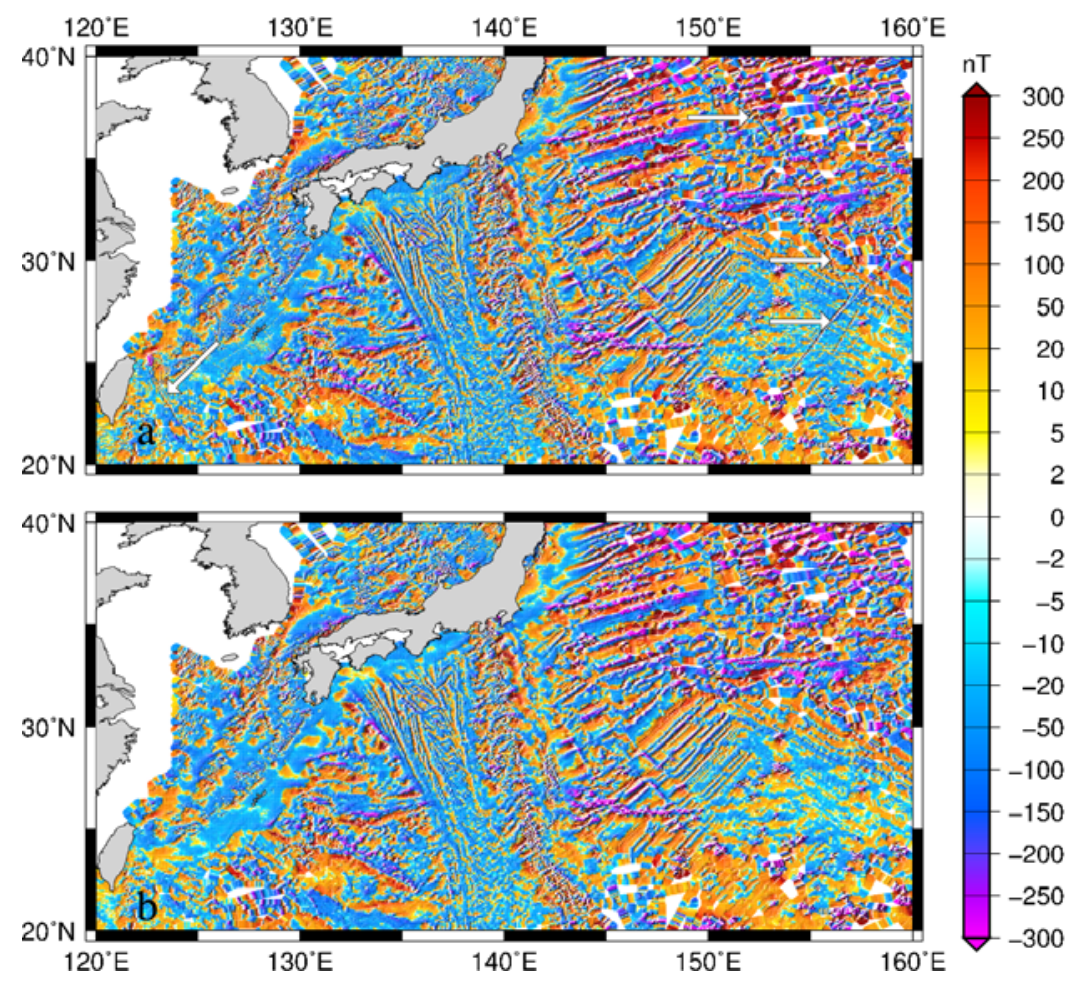

Figure 11. Gridded magnetic anomaly maps (with illumination from southeast) over the northwestern part of the Pacific Ocean (a) before and (b) after the leveling phase. Straight, sometimes $10^{\circ}$-long, anomalous features indicated by arrows on the eastern part of Figure 11a, as well as more localized ones in the southwestern part, are removed by this leveling.

for $r_{0}$ was suitable (larger values did not improve the $\left.\mathrm{RMS}_{\mathrm{COD}}\right)$. Additionally, leveling effects of nearby track lines without crossovers were also included by using this function. For instance, data offsets in a detailed survey with parallel tracks at $5 \mathrm{~km}$ intervals (or less) were significantly reduced.

[38] As described in section 5, two Gaussian high-pass filtering steps were applied. First, the full width of the filter was set to $1000 \mathrm{~km}$ to reduce long-wavelength offsets. If crossovers at $50 \mathrm{~km}$ intervals (or less) existed along a track line, the offsets could be reduced by applying only one filtering step with a shorter full width to minimize the CODs with other track lines. However, crossovers are heterogeneously distributed over the oceans. Some track lines, particularly in the southern oceans, had no crossovers for $500 \mathrm{~km}$ intervals (or more). In such cases, the high-pass filtering with a full width as large as $1000 \mathrm{~km}$ was the only method available to reduce the long-wavelength offsets. For offsets at shorter wavelengths, a second step using $100 \mathrm{~km}$ as the filter full width was applied. The problem was to keep short-wavelength information in the NGDC-720-reduced data set (containing mostly wavelengths shorter than $50 \mathrm{~km}$ ). Some components shorter than $50 \mathrm{~km}$ could be lost if the full width was smaller than $50 \mathrm{~km}$. Using a $100 \mathrm{~km}$ filter, the effect was not so serious. Therefore, a Gaussian high-pass filter with a full width of $100 \mathrm{~km}$ was probably the best to reduce short-wavelength offsets.

[39] Other leveling methods exist. For aeromagnetic surveys, tie lines perpendicular to the main flight lines associated to a regular constant sampling rate allow an accurate leveling of data. For irregular and widespread data as marine magnetics, the leveling approach has to consider all neighboring values (i.e., those of nearby cruises as well as those of the studied cruise). Such methods are rarely described in the literature. In the approach developed by $H s u$ [1995], a weight for each track is first determined by the inverse of the variance of all its CODs. The corrected value at each COD is also defined by the weighted average of values along the two tracks with previously determined weights. Corrections of all data could be calculated by interpolation/extrapolation of these corrections at crossover points. Although this method could be applied as an alternative of ours, it is crucial to avoid too many variations in the data set, particularly where the anomaly values may change rapidly near a crossover point with large COD and where two or more CODs are very close. In this sense, our method is more convenient than the Hsu's [1995] approach since it avoids too large variations of corrections by introducing a low-pass filtering step. Data accuracy of each cruise was not considered, i.e., the weight only depends on the distance from the considered point. Inclusion of cruise weights, as well as anisotropic effects, might improve our leveling process.

\section{Conclusions}

[40] Each magnetic measurement stored in the GEODAS data set was accurately corrected, not only by subtracting the reliable core and external field contributions, but also by checking the anomaly value track by track. Additionally, a leveling phase was performed to build a coherent magnetic 


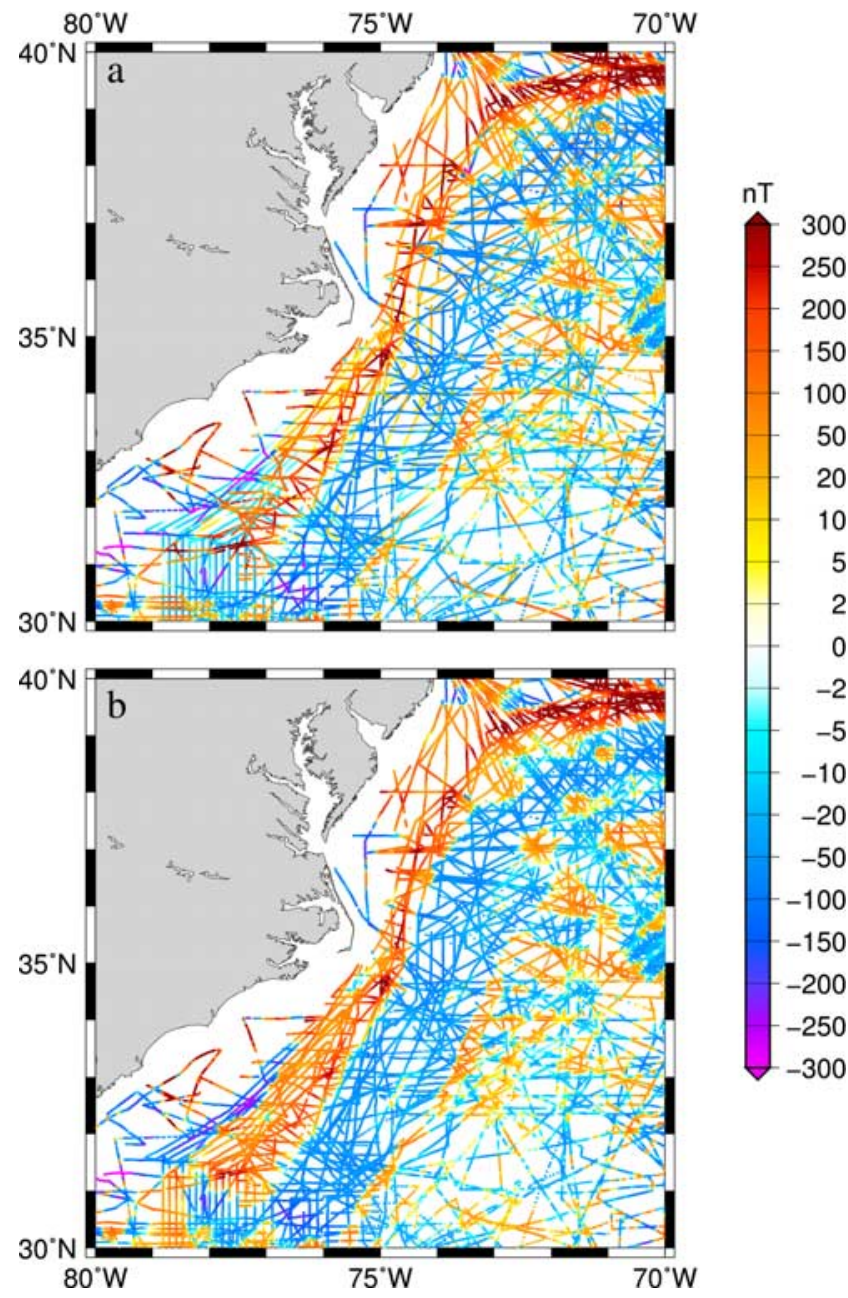

Figure 12. Magnetic anomaly maps over the western part of the North Atlantic Ocean (a) before and (b) after the leveling phase. Numerous offsets between overlapping subsets are shown on Figure 12a, especially in the southwestern part, while Figure $12 \mathrm{~b}$ indicates that offsets disappeared after leveling.

map over oceanic areas. At crossovers, the RMS of the differences was reduced from $179.6 \mathrm{nT}$ (original data) to $35.9 \mathrm{nT}$ (cleaned and leveled data).

[41] This procedure is not only a first-class tool for the whole scientific community that uses marine magnetic data, but it should also enhance the quality of the next version of the magnetic anomaly map of the world (WDMAM project). However, the acquisition of magnetic data is still needed, particularly in the southern oceans. Therefore, we encourage teams or institutions to perform marine magnetic surveys and to forward the data to GEODAS and/or to contact the authors. Furthermore, any references of existing regional marine magnetic databases (or maps) not reported in GEODAS would be greatly helpful in order to check our resulting map (by comparison), to incorporate these data into our set to improve the quality of our map and to eliminate some gaps. The final CM4-corrected cleaned and leveled data sets are now available online at ftp:// WDMAM2008@ftp.gtk.fi (request to J. Korhonen for login and password).

[42] Acknowledgments. The authors would like to thank the Associate Editor P. Taylor and two anonymous reviewers for their constructive comments. Ingo Wardinski, Vincent Lesur, Francisca Staines-Urias, and Christine Pailles are gratefully acknowledged for their preliminary reviews, as well as Juha Korhonen, Mioara Mandea, Mohamed Hamoudi, Erwan Thébault, Aude Chambodut, and the WDMAM executive committee for their support. We are also indebted to people involved in the acquisition and storage (GEODAS) of marine magnetic data of the world. For this study, Y.Q. was funded by the German Science Foundation DFG. All figures and crossover calculations were performed using the GMT software [Wessel, 1989; Wessel and Smith, 1998].

\section{References}

Bullard, E., and R. Mason (1961), The magnetic field astern of a ship, Deep Sea Res., 8, 20-27.

Chandler, M. T., and P. Wessel (2008), Improving the quality of marine geophysical track line data: Along-track analysis, J. Geophys. Res., 113, B02102, doi:10.1029/2007JB005051

Hsu, S.-K. (1995), A cross-over technique to adjust track data, Comput. Geosci., 21, 259-271.

Korhonen, J., et al. (2007), Magnetic anomaly map of the world, scale 1:50,000,000, 1st ed., Comm. for the Geol. Map of the World, UNESCO, Paris.

Macmillan, S., and S. Maus (2005), International Geomagnetic Reference Field-the tenth generation, Earth Planets Space, 57, 1135-1140.

Maus, S. (2008), The geomagnetic power spectrum, Geophys. J. Int., 174, 135-142, doi:10.1111/j.1365-246X.2008.03820.x.

Maus, S., H. Lhr, M. Rother, K. Hemant, G. Balasis, P. Ritter, and C. Stolle (2007a), Fifth-generation lithospheric magnetic field model from CHAMP satellite measurements, Geochem. Geophys. Geosyst., 8, Q05013, doi:10.1029/2006GC001521.

Maus, S., T. Sazonova, K. Hemant, J. D. Fairhead, and D. Ravat (2007b), National Geophysical Data Center candidate for the World Digital Magnetic Anomaly Map, Geochem. Geophys. Geosyst., 8, Q06017, doi:10.1029/2007GC001643

Metzger, D., and J. Campagnoli (2007), Marine Trackline Geophysics Data on DVD, version 5.0.10, Natl. Geophys. Data Cent., Boulder, Colo.

Olsen, N., H. Lühr, T. Sabaka, M. Mandea, M. Rother, L. Toffner-Calusen, and S. Choi (2006), CHAOS - A model of the Earth's magnetic field derived from CHAMP, Oersted, and SAC-C magnetic satellite data, Geophys. J. Int., 166, 67-75.

Sabaka, T., N. Olsen, and M. Purucker (2004), Extending comprehensive models of the Earth's magnetic field with Oersted and CHAMP data, Geophys. J. Int., 159, 521-547.

Sapunov, V., A. Denisov, O. Denisova, and D. Saveliev (2001), Proton and Overhauser magnetometers metrology, Contrib. Geophys. Geodesy, 31(1), 119.

Smith, W., and D. Sandwell (1997), Global seafloor topography from satellite altimetry and ship depth soundings, Science, 277, 1956-1962.

Thakur, N., T. Gangadhara Rao, C. Subrahmanyam, and R. Khanna (1999), Crossover analysis of geophysical data in Bay of Bengal, Geo Mar. Lett., $19,262-269$.

Váczyová, M., and Z. Vörös (2001), Result of Diflux and proton magnetometer comparison at the IXth IAGA Workshop, Contrib. Geophys. Geod., 31(1), 417.

Wessel, P. (1989), XOVER: A cross-over error detector for track data, Comput. Geosci., 15, 333-346.

Wessel, P., and W. Smith (1998), New, improved version of Generic Mapping Tools released, Eos Trans. AGU, 79(47), 579.

Wessel, P., and A. Watts (1988), On the accuracy of marine gravity measurements, J. Geophys. Res., 93, 393-413.

M. Catalán, Geophysics Department, Real Instituto y Observatorio de la Armada, San Fernando E-11100, Spain. (mcatalan@roa.es)

T. Ishihara, Institute of Geology and Geoinformation, Geological Survey of Japan, AIST AIST Central 7, Tsukuba, Ibaraki, 305-8567 Japan. (t-ishihara@aist.go.jp)

Y. Quesnel, CEREGE, Europôle de l'Arbois, BP80, F-13545 Aix-enProvence CEDEX 04, France. (quesnel@cerege.fr) 